\title{
General Psychiatry Altered heartbeat perception sensitivity associated with brain structural alterations in generalised anxiety disorder
}

\author{
Hui Li, ${ }^{1}$ Bin Zhang, ${ }^{2}$ Qiang Hu, ${ }^{1}$ Lanlan Zhang, ${ }^{3}$ Yi Jin, ${ }^{4}$ Jijun Wang, ${ }^{1}$ Huiru Cui, ${ }^{1}$ \\ Jiaoyan Pang, ${ }^{5,6}$ Chunbo Li ${ }^{1}$
}

To cite: Li H, Zhang B, Hu Q, et al. Altered heartbeat perception sensitivity associated with brain structural alterations in generalised anxiety disorder. General Psychiatry 2020;33:e100057. doi:10.1136/ gpsych-2019-100057

$\mathrm{HL}$ and $\mathrm{BZ}$ contributed equally.

Received 27 January 2019 Revised 08 0ctober 2019 Accepted 28 November 2019

A) Check for updates

(c) Author(s) (or their employer(s)) 2020. Re-use permitted under CC BY-NC. No commercial re-use. See rights and permissions. Published by BMJ.

For numbered affiliations see end of article.

Correspondence to

Dr Chunbo Li; licb@smhc.org.cn

\section{ABSTRACT}

Background Palpitation is a common complaint in generalised anxiety disorder (GAD). Brain imaging studies have investigated the neural mechanism of heartbeat perception in healthy volunteers. This study explored the neuroanatomical differences of altered heartbeat perception in patients with GAD using structural MRI. Aims Based on the strong somatic-interoceptive symptoms in GAD, we explored the regional structural brain abnormalities involved in heartbeat perception in patients with GAD.

Methods This study was applied to the a priori regions using neuroanatomical theories of heartbeat perception, including the insula, anterior cingulate cortex, supplementary motor area and prefrontal cortex. A total of 19 patients with GAD and 19 healthy control subjects were enrolled. We used the FMRIB Software Library voxel-based morphometry software for estimating the grey matter volume of these regions of interest and analysed the correlation between heartbeat perception sensitivity and the volume of abnormal grey matter.

Results Patients with GAD showed a significantly decreased volume of grey matter in their left medial prefrontal cortex, right orbital frontal cortex and anterior cingulate cortex. The grey matter volume of the left medial prefrontal cortex negatively correlated with heartbeat perception sensitivity in patients with GAD.

Conclusions It should be the first study that shows heartbeat perception is associated with brain structure in GAD. Our findings suggest that the frontal region may play an important role in aberrant heartbeat perception processing in patients with GAD, and this may be an underlying mechanism resulting in the abnormal cardiovascular complaints in GAD. This is hypothesised as a 'top-down' deficiency, especially in the medial prefrontal cortex. This will provide the foundation for a more targeted region for neuromodulation intervention in the future.

\section{INTRODUCTION}

Generalised anxiety disorder (GAD) is a common, serious psychiatric condition, affecting about $2 \%-6 \%$ of the general population during their lifetime. ${ }^{1}$ Patients with GAD may experience cardiovascular complaints such as a rapid heartbeat, palpitation and feeling of impending death. ${ }^{2}$ They frequently seek treatment in primary and specialty healthcare settings resulting in substantial personal, societal and economic costs.

Generally, the most familiar signal from the cardiovascular system is a heartbeat, which is more easily felt than other bodily sensations. ${ }^{3-6}$ Schandry et al first used a mental tracking procedure to measure the heartbeat perception score (HPS) to assess the accuracy of heartbeat perception. ${ }^{7}$ Previous study showed those patients with GAD that had greater cardiovascular symptoms also had higher levels of cardiac lability and a heightened sensitivity to bodily responses than nonanxious individuals. A review by Van der Does et al showed individuals with anxiety disorders perceived their heartbeat more accurately than healthy controls (HC). ${ }^{8}$ Furthermore, interoceptive awareness was frequently used to assess their heartbeat perception, and previous neuroimaging studies have investigated the neural mechanism associated with heartbeat perception in healthy individuals. ${ }^{9}$

Neuroimaging techniques have been applied to investigate the neural mechanism associated with heartbeat perception. Critchley et al measured regional brain activity using functional MRI (fMRI) during an interoceptive task and found that this activated the bilateral insular, somatomotor, parietal cortices and anterior cingulate cortices (ACC).$^{10}$ Pollatos et al investigated interoceptive awareness and feelings in healthy male subjects, and found that interoceptive awareness is related to enhanced activation in the insula, somatosensory cortices, ACC and prefrontal cortices. ${ }^{11}$ Additionally, a study showed that the insula and ACC are critical for awareness of heartbeat sensations and helped mediate somatosensory afferents from the skin. ${ }^{9}$ Critchley et al used voxel-based morphometry (VBM) to 
explore the relationship between the regional grey matter volume (GMV) and accuracy on the heartbeat detection task, and found GMV in the right anterior insula, orbitofrontal cortex (OFC) and midline cerebellum correlated with performance on the heartbeat detection task. ${ }^{10}$ Several regions are associated with heartbeat perception, including the insula, ACC, supplementary motor area (SMA) and prefrontal cortices. However, all of these studies investigated a healthy population, and the function and structure of these regions are unclear in patients with GAD which have shown abnormal heartbeat perception.

Recently, several structural MRI studies have investigated the brain structure of patients with GAD. Moon $e t$ $a l$ showed that patients with GAD showed a significantly reduced GMV in their hippocampus, midbrain, thalamus, insula and superior temporal gyrus as compared with that in HCs. ${ }^{12}$ However, another study by Strawn $e t ~ a l^{13}$ found that adolescents with GAD had increased GMV in their right precuneus and right precentral gyrus but decreased GMV in their left orbital gyrus and posterior cingulate. In the most recent MRI studies involving patients with GAD, abnormal cortical thickness in the right hemisphere appears to be involved in the illness, but no significant alterations in cortical surface area and GMV were observed. ${ }^{14}$ Maron and Nutt summarised the results of structural MRI studies and described the anatomical changes in the brain, particularly within regions related to anxiety neurocircuitry, including the prefrontal region. ${ }^{15}$ As the findings of structural MRI in GAD are inconsistent, these findings combined with well-developed neuroanatomical theories of heartbeat perception in healthy individuals can confirm the regions of interest (insula, ACC, SMA and prefrontal cortex (PFC)). Therefore, we speculate the following hypotheses: (A) patients with GAD may show an altered GMV in the regions related to heartbeat perception; (B) these altered brain structures would significantly correlate with heartbeat perception as evaluated using a mental tracking procedure; (C) cardiovascular symptoms correlated with these abnormal brain regions in patients with GAD would be found.

\section{SUBJECTS AND METHODS}

\section{Subjects}

Nineteen subjects with GAD (6 males and 13 females) and 19 age and gender-matched HC subjects (7 males and 12 females) without any psychiatric illnesses were studied. GAD subjects were recruited from the outpatient clinic of the Shanghai Mental Health Center. HCs were recruited from among hospital staff and through advertisements. They received a small remuneration for their participation. One expert clinician (CL) confirmed the diagnoses based on the Diagnostic and Statistical Manual of Mental Disorders-Fourth Edition (DSM-IV) criteria for $\mathrm{GAD}^{16}$ and the diagnoses were further checked by two research doctors ( $\mathrm{QH}$ and LZ) using a Structured Clinical Interview from the DSM-IV Patient Edition (SCID-I/P). ${ }^{17}$ Inclusion criteria were: (1) aged between 18 and 60 years; (2) a score on the Hamilton Anxiety Rating Scale (HAMA) ${ }^{18}$ greater than 14, and a score on the Hamilton Depression Rating Scale (HAMD) ${ }^{19}$ less than 14; and (3) have completed at least 6 years of primary education. Exclusion criteria were head trauma leading to a loss of consciousness, substance abuse or substance dependence disorder, contraindication to MR scanning and a history of other mental illnesses. The SCID Non-Patient Edition (SCID-I/NP) was administered to all HC subjects to exclude subjects with an axis I or axis II psychiatric disorder (figure 1).

Before their MRI examination, every participant completed questionnaires covering their demographic data and assessing their anxiety levels through a HAMA

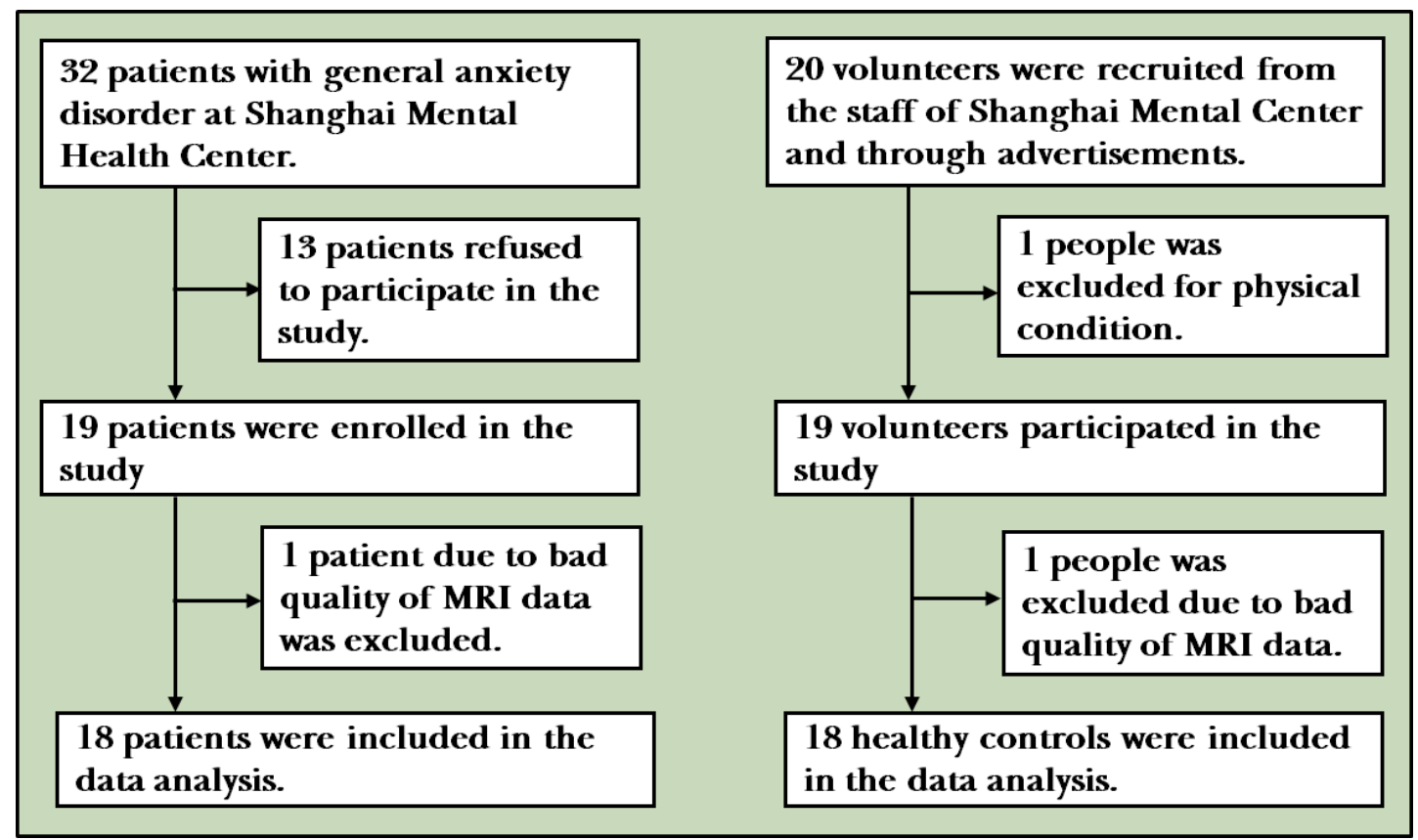

Figure 1 The flow chart of the study. 
and 17-question HAMD. HAMA is a clinician-administered scale that assesses anxiety symptoms through two subscales: the anxiety subscale and the somatic anxiety subscale. Item 9 reflects anxiety-related cardiovascular symptoms, and the severity of this is determined using a 5-point scale $(0=$ not present, $4=$ severe $)$. All participants signed a written informed consent form before study participation.

\section{Methods}

Altered heartbeat perception sensitivity mainly manifested in two aspects of the population with anxiety. ${ }^{20}$ One was high levels of cardiovascular symptoms such as a rapid heartbeat or palpitation. Item 9 of the HAMA scale was used to evaluate the anxiety-related cardiovascular symptoms in this study. ${ }^{18}$ The other was assessed using a behavioural measurement, such as the mental tracking paradigm.

\section{Mental tracking paradigm}

This paradigm has been developed and evaluated for the assessment of heartbeat perception. ${ }^{21}$ It offers the most promising results in distinguishing clinical and nonclinical anxiety groups. All subjects were required to pay attention to their heartbeat and count their heartbeats silently. The number of heartbeats counted was then reported by the patients at the end of the experiment. During this period, a portable electrocardiograph was used to record the occurrence of $\mathrm{R}$ waves so that the exact number of heartbeats could be determined. The experiment was conducted three times, and counts were made at 26, 21 and $36 \mathrm{~s}$ randomly. After the entire experiment, data were calculated using the following formula. The HPS was used to measure the accuracy of heartbeat perception, with a full score of 1 . Higher scores indicated a higher heartbeat perception level. ${ }^{22}$

$$
P=\frac{1}{k} \sum_{i=1}^{k}\left(1-\frac{|O i-G i|}{\mathrm{O} i}\right)
$$

where $P$ showed the HPS; ' $i$ ' was the number of times for the experiment; $O$ was the actual number of times for the subjects' heartbeat during the experiment; and $G$ was the number of heartbeats counted by the subjects during the experiment period.

\section{MRI acquisition}

For each participant, a 3D-T1 image was obtained with a 3 T Magnetom Trio Tim scanner (Siemens, Erlangen, Germany). Structural images were acquired using a magnetisation prepared rapid gradient echo sequence with the following parameters: echo time $=2.46 \mathrm{~ms}$, repetition time $=1900 \mathrm{~ms}$, field of view= $240 \mathrm{~mm} \times 240 \mathrm{~mm}$, flip angle $9^{\circ}$, bandwidth $140 \mathrm{~Hz} /$ pixel, acquisition matrix $256 \times 256 \times 192$, voxel size $1 \times 1 \times 1 \mathrm{~mm}^{3}$.

\section{MRI data preprocessing}

T1-weighted structural data were analysed with FSL-VBM V.1.1 (http://fsl.fmrib.ox.ac.uk/fsl/fslwiki/FSLVBM), an optimised VBM protocol ${ }^{23}$ carried out with FSL tools. First, structural images were brain extracted and grey matter (GM) segmented before being non-linearly registered to the Montreal Neurological Institute (MNI) 152 standard space using non-linear registration. The resulting images were averaged and flipped along their $\mathrm{x}$-axis to create leftright symmetry and a study-specific GM template. Second, all native GM images were non-linearly registered to this study-specific template and modulated to correct for local expansion due to the non-linear component of the spatial transformation. The modulated GM images were then smoothed with an isotropic Gaussian kernel with a sigma of $8 \mathrm{~mm}$. In this study region of interests (ROI) included the ACC, bilateral insula, SMA and PFC, as they are associated with interoceptive awareness. These ROI masks were derived from the WFU-Pickatlas ${ }^{24}$ and were created based on the Talairach Daemon in the MNI space.

\section{Statistical analysis}

To analyse the demographic and clinical characteristics, we used the Statistical Package for the Social Science V.19.0 (SPSS). A paired t-test was performed to evaluate the differences in age, educational level, HAMA, HAMD and HPS between patients with GAD and HCs. A Wilcoxon signed-rank test was used to compare sex. All significance levels were set at $\mathrm{p}<0.05$.

A voxel-wise general linear model was applied using permutation-based non-parametric testing to build statistical maps $(\mathrm{p}<0.05)$ between patients with GAD and controls for VBM data. Significant clusters from these statistical maps were used in the small volume correction (SVC) analysis to restrict the comparisons to specific voxels of ROI. In the analyses, total GMVs, sex and age were considered as covariates.

To identify the association between structural abnormalities and heartbeat perception sensitivity, the average GMV in abnormal areas was extracted. The relationship between abnormal regions and heartbeat perception sensitivity was determined using the Spearman correlation analysis $(\mathrm{p}<0.05)$.

\section{RESULTS}

\section{Demographic and clinical characteristics}

One subject with GAD and one healthy individual were excluded due to the insufficient quality of their MRI data. Eighteen patients with GAD and 18 HCs were analysed. In those patients with GAD, 13 used at least one type of medication, and five patients did not take any medicine. The patient group and control group had no significant differences in age, gender, educational level and HPS scores. There were significant differences in the HAMA and HAMD scores between patients with GAD and the control group (table 1).

\section{Regional differences in GMV}

In the SVC analysis, patients with GAD demonstrated a significantly decreased GMV in their left medial prefrontal cortex (MPFC), ACC and right OFC, as compared with those in HCs (table 2 and figure 2, corrected $\mathrm{p}<0.05$ ).

\section{Correlations between regional GMV and HPS}

The GMV of the left MPFC was negatively correlated with HPS $(\mathrm{r}=-0.41, \mathrm{p}=0.05)$ in patients with GAD (figure 3 ). 
Table 1 Demographic and clinical characteristics for participants

\begin{tabular}{lllcc}
\hline Characteristic & GAD $(\mathbf{n}=18)$ & HC $(\mathbf{n}=18)$ & $\boldsymbol{t} / \mathbf{Z}$ & P value \\
\hline Age (years) & $41.89(10.79)$ & $38.17(10.56)$ & 0.99 & 0.34 \\
Sex (male/female) & $13 / 5$ & $11 / 7$ & 0.71 & 0.48 \\
Educational level (years) & $13.44(2.85)$ & $16.06(2.93)$ & 1.78 & 0.09 \\
HAMA & $18.80(9.36)$ & $0.78(0.94)$ & 19.59 & $<0.001$ \\
HAMD & $8.89(5.32)$ & $0.94(1.26)$ & 5.18 & $<0.001$ \\
HPS & $0.61(0.15)$ & $0.68(0.23)$ & 1.00 & 0.33 \\
\hline
\end{tabular}

GAD, generalised anxiety disorder; HAMA, Hamilton Anxiety Rating Scale; HAMD, Hamilton Depression Rating Scale; HC, healthy control; HPS, heartbeat perception score.

However, in the control group, there was no significant relationship between the GMV of the left MPFC and HPS $(\mathrm{r}=-0.07, \mathrm{p}=0.80)$. There was no significant relationship between the GMV of the ACC or right OFC and HPS in both groups.

\section{Correlations between regional GMV and cardiovascular symptoms}

To examine the clinical relevance of our findings, exploratory correlation analyses were also conducted between the GMV of abnormal ROI and the patients' cardiovascular symptoms score. We found a significant association between the GMV of the left MPFC and cardiovascular symptoms in the GAD group $(\mathrm{r}=-0.57, \mathrm{p}=0.01)$, which indicates that a smaller GMV of the MPFC reflects higher levels of cardiovascular anxiety.

\section{DISCUSSION}

\section{Main findings}

This study had two main findings. First, the GMVs in the MPFC, ACC and OFC were significantly decreased in patients with GAD as compared with the HC group. The volume in the MPFC was seen to be related to heartbeat sensitivity in patients with GAD but not in the healthy population. ${ }^{25}$ There are few studies that have examined the relationship between structural MRI and cardiac perception in the healthy population. To our knowledge, it should be the first study to explore the neural structures involved in processing enhanced cardiac perception in patients with GAD. Our results showed that heartbeat perception in patients with GAD mainly involved the left MPFC, ACC and right OFC, implicating an abnormal top-down control. There was a significant correlation between the GMV of the left MPFC and heartbeat perception sensitivity in GAD subjects as evaluated by behavioural measurement and cardiovascular symptoms. Hence, the MPFC brain region may significantly contribute to abnormal heartbeat-related information regulation processes in patients with GAD.

This study aimed to explore the neuroanatomical evidence of abnormal heartbeat perception in patients with GAD. The brain regions found in this study are consistent with previous fMRI studies, which have shown abnormal activations in the PFC, OFC and ACC which help regulate emotion, such as fear generalisation and threat inhibition, worry and anxiety in patients with GAD. ${ }^{26} 27$ Previous studies showed that the activation of the MPFC is decreased during emotional judgements, fear inhibition and the successful evaluation of threat and safety. Neuroimaging results in rats have explored the neurobiology mechanism underlying GAD and have shown a dysfunctional MPFC, inhibition of the OFC and amygdala hyperactivity during the processing of potentially threatening environmental information. ${ }^{28}$ Furthermore, Kujawa et $a l$ s study showed that the activation of the ACC involved in the processing of threatening faces could predict the effects of treatment with selective serotonin reuptake inhibitors and cognitive-behavioural therapy in children and adolescents with anxiety disorder, ${ }^{29}$ which suggests that characteristic functional abnormality of the ACC may exist in patients with GAD. Taken together, neuroanatomical evidence from this study and previous fMRI studies suggest that patients with GAD showed greater dysfunction in their emotional regulation and heartbeat perception in these three regions. However, no further analysis was performed to explore the relationship between emotional regulation and heartbeat perception in this study. These connections require further confirmation, and the mechanisms that may exist in patients with GAD should be explored in future studies, although a study of the normal population may indicate that there is a correlation between interoceptive judgements in the emotional assessment.

Table 2 Regional differences in grey matter

\begin{tabular}{lccccc}
\hline Region & \multicolumn{1}{c}{ MNI coordinates } & & Voxels, n & P value \\
\hline Right orbital frontal cortex & 2 & 44 & 32 & 40 & 0.01 \\
Left medial prefrontal cortex & -4 & 30 & 52 & 35 & 0.03 \\
Anterior cingulate cortex & -2 & 30 & 50 & 55 & 0.02 \\
\hline
\end{tabular}




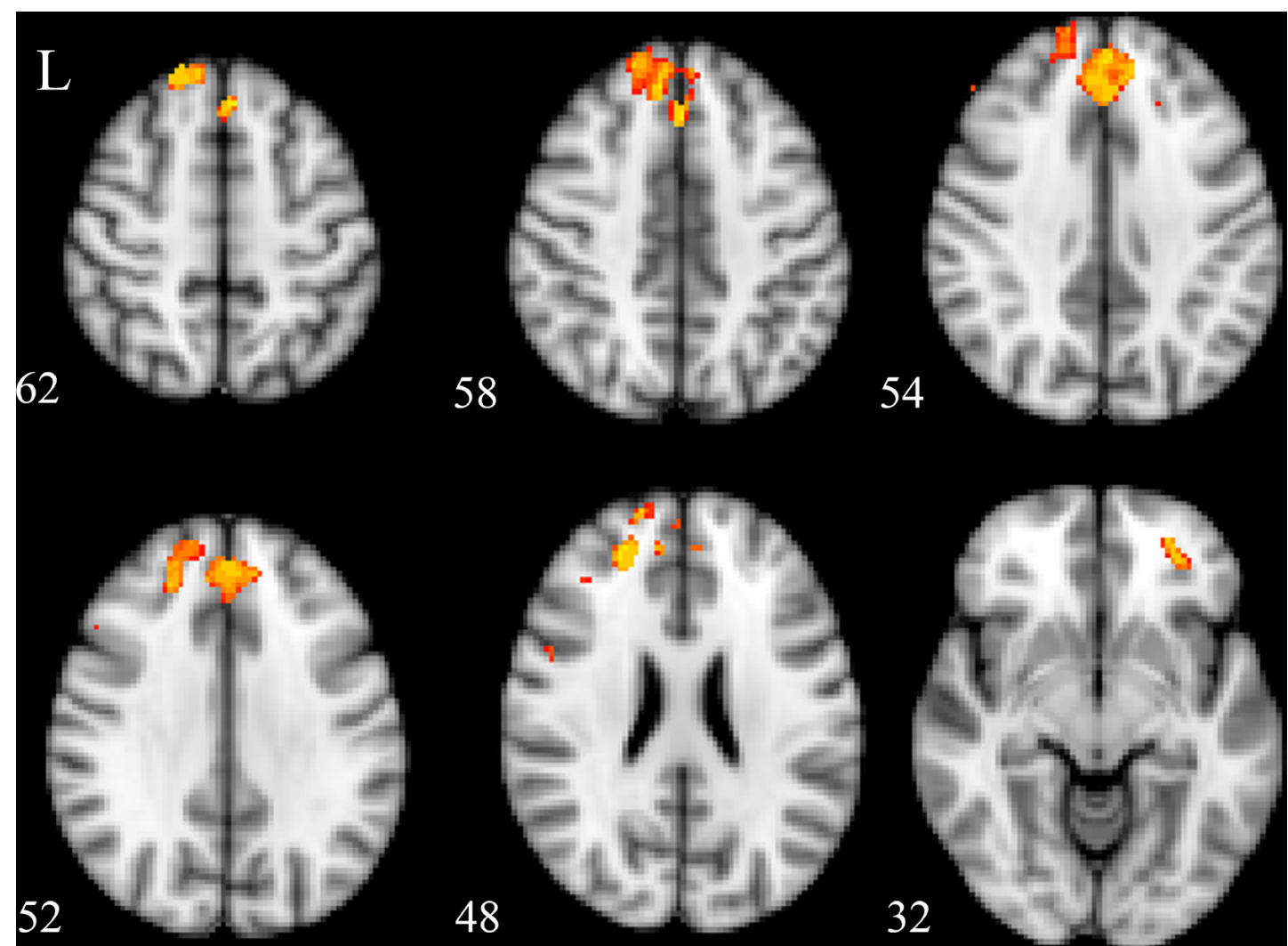

Figure 2 Clusters that showed a significant difference between patients with generalised anxiety disorder (GAD) and controls.

Recently, neuroimaging studies have investigated the neural mechanism associated with interoception in healthy individuals and have suggested the functional neural substrates active in interoceptive judgements are indeed the same as emotional assessments. Previous studies have shown that interoceptive awareness is related to neural activity in the insula, somatosensory cortices, ACC and prefrontal cortices. ${ }^{30}$ The insula, a region involved in heartbeat perception processing circuits, ${ }^{32}$ was not seen in this current study. Critchley et al found that the right anterior insula cortex was the most accurate in making timing discriminations that reflected the

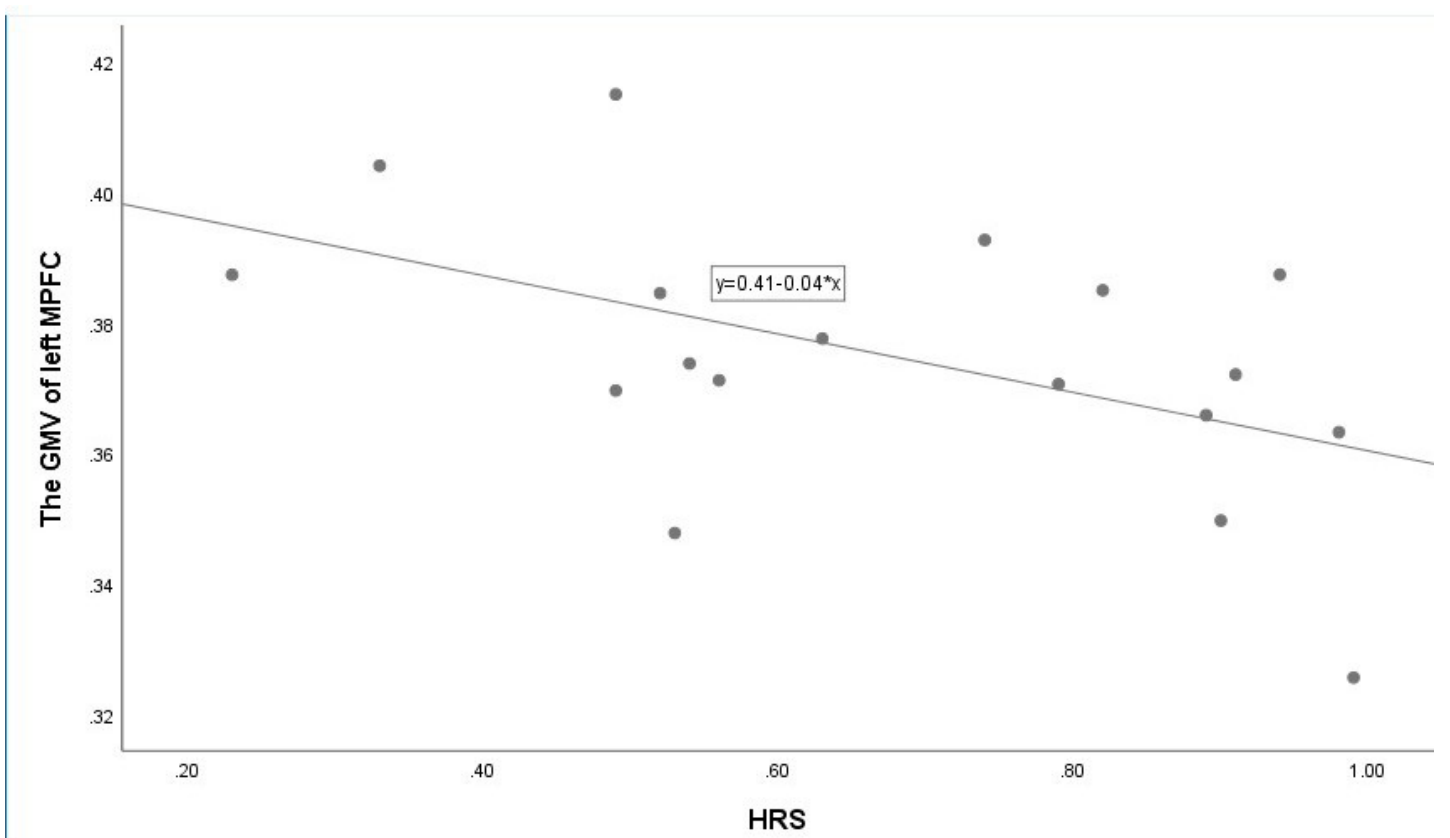

Figure 3 Correlation between grey matter volume (GMV) of left medial prefrontal cortex (MPFC) and score on heartbeat perception score (HPS) in patients with generalised anxiety disorder (GAD). 
heartbeat and that the extent of activity in this region correlates with self-rated body awareness. However, Critchley's studies used a healthy population. We cannot definitively conclude this, since it may also be a top-down abnormality, mainly in the PFC, not in the insular.

This study examined interoceptive awareness-related cortexes with an ROI-based method and revealed a reduction in the volume of the MPFC, ACC and OFC in patients with GAD. In line with our study, Shang et al performed a meta-analysis of the VBM studies done regarding anxiety disorder to estimate the differences between patients and controls in terms of clusters of significant GM. They found that the atrophy of the ventral ACC and medial PFC generated a dysfunctionally top-down control, which was the main reason for the occurrence of anxiety disorders. ${ }^{33}$ However, the volumetric alterations in patients with GAD were inconsistent between the studies. Terlevic et al showed reduced hypothalamic volumes using a manually traced hypothalamus in patients with GAD, ${ }^{34}$ and a decrease in hippocampal volumes was found in another study. Makovac et $a l^{35}$ reported deficits of GMV in the supramarginal, precentral and bilaterally postcentral gyrus in patients with GAD. Liao et $a l^{36}$ found significantly increased GMV of the right putamen in patients with GAD as compared with a healthy group. Moon and Jeong conducted a series of research and found significant volume reductions in the hippocampus, midbrain, thalamus, insula and superior temporal gyrus in patients with GAD. ${ }^{37}$ The inconsistent results are possibly due to the different styles in evaluating GM volumetric alterations. Some studies focused on specific brain ROIs, and further analysis of the relationships between the ROI brain areas and clinical characters is needed. Others analysed GMV changes in the whole-brain region between patients with GAD and HCs using VBM. In this current study, we investigated regional GMV in patients with GAD and applied a priori region from well-developed neuroanatomical theories of heartbeat perception. We found abnormal perception in the left MPFC, ACC and right OFC, implicating a top-down control. Furthermore, there was a significant correlation between the GMV of the left MPFC and heartbeat perception sensitivity in patients with GAD, suggesting that MPFC constitutes a highly important structure in the pathogenesis of GAD, possibly via the mediation of interoceptive processes.

\section{Limitations}

This study has several potential limitations that should be considered. The sample size was less than the number needed to reach significance at a behavioural level. Although we found that an abnormal brain structure was related to interoceptive processing in patients with GAD, a larger sample is needed to test this conclusion. Previous research reported that the assessment of heartbeat perception in a solely behavioural or selfreporting questionnaire-based manner is not ideal, and indicated that heartbeat-evoked brain potentials (HEP), an electrocortical potential time locked to the cardiac $\mathbf{R}$ wave, might be a more objective quantitative assessment tool. ${ }^{25}$ It was proposed to use HEP to evaluate heartbeat perception in future studies. Further limitations are that some patients with GAD received different treatments, which is a well-recognised issue in similar brain structure studies.

\section{Implications}

In summary, this is the first study to report decreased GMV in MPFC, ACC and OFC in patients with GAD, and that the changes in the MPFC are related to heartbeat sensitivity. These findings suggest that the frontal region may play an important role in aberrant heartbeat perception processing in patients with $\mathrm{GAD}$, and this may be the mechanism underlying abnormal cardiovascular complaints associated with GAD. This is hypothesised as a 'top-down' deficiency, especially in the MPFC, which will provide the foundation for more targeted neuromodulation intervention regions in the future. The primary focus of this study was to evaluate the characteristic structural abnormalities in patients with GAD and find an abnormal brain structure related to interoceptive processing. However, functional imaging assessments were not used. Future studies should more comprehensively analyse this using both the structure and function of the brain.

\section{Author affiliations}

${ }^{1}$ Shanghai Mental Health Center, Shanghai Jiao Tong University School of Medicine, Shanghai, China

${ }^{2}$ Guangdong Engineering Technology Research Center for Translational Medicine of Mental Disorders, The Affiliated Brain Hospital of Guangzhou Medical University (Guangzhou Huiai Hospital), Guangzhou, China

${ }^{3}$ Department of Psychiatry, Suzhou Guangji Hospital, Suzhou, China

${ }^{4}$ Institute of Psychology, Chinese Academy of Sciences, Shanghai, China

${ }^{5}$ Department of Psychology, Shanghai University of Political Science and Law, Shanghai, China

${ }^{6}$ Shanghai Key Laboratory of Psychotic Disorders, Shanghai Mental Health Center, Shanghai Jiao Tong University School of Medicine, Shanghai, China

Contributors $\mathrm{CL}$ and JW designed the project. BZ, $\mathrm{HL}, \mathrm{QH}, \mathrm{LZ}$ and $\mathrm{HC}$ performed the experiment. YJ analysed the MRI data and interpreted the results. $\mathrm{HL}$ and BZ wrote the manuscript. JP contributed to manuscript editing. CL handled the work of revising the manuscript. All authors have read and approved the final version of the manuscript.

Funding This work was supported by Shanghai Science and Technology Commission (16411965000; 18411952400), Shanghai Jiao Tong University Foundation (YG2016MS37), National Key R\&D Program of China (2018YFC2001605, 2019YFA0706200), Natural Science Foundation of Shanghai (18ZR1432600, 81071098), Shanghai Municipal Commission of Health and Family Planning Foundation (20164Y0215).

Competing interests None declared.

Patient consent for publication Not required.

Provenance and peer review Not commissioned; externally peer reviewed.

Data availability statement No data are available. The data used to support the findings of this study have not been made available.

Open access This is an open access article distributed in accordance with the Creative Commons Attribution Non Commercial (CC BY-NC 4.0) license, which permits others to distribute, remix, adapt, build upon this work non-commercially, and license their derivative works on different terms, provided the original work is properly cited, appropriate credit is given, any changes made indicated, and the use is non-commercial. See: http://creativecommons.org/licenses/by-nc/4.0/. 


\section{REFERENCES}

1 Comer JS, Blanco C, Hasin DS, et al. Health-Related quality of life across the anxiety disorders: results from the National epidemiologic survey on alcohol and related conditions (NESARC). J Clin Psychiatry 2011;72:43-50.

2 Hoehn-Saric R, McLeod DR, Funderburk F, et al. Somatic symptoms and physiologic responses in generalized anxiety Disorderand panic disorder. Arch Gen Psychiatry 2004;61:913-21.

3 Domschke K, Stevens S, Pfleiderer B, et al. Interoceptive sensitivity in anxiety and anxiety disorders: an overview and integration of neurobiological findings. Clin Psychol Rev 2010;30:1-11.

4 Petzschner FH, Weber LA, Wellstein KV, et al. Focus of attention modulates the heartbeat evoked potential. Neuroimage 2019;186:595-606.

5 Yoris A, Esteves S, Couto B, et al. The roles of interoceptive sensitivity and metacognitive interoception in panic. Behav Brain Funct 2015;11.

6 Khalsa SS, Adolphs R, Cameron OG, et al. Interoception and mental health: a roadmap. Biol Psychiatry 2018;3:501-13.

7 Schandry R, Bestler M, Montoya P. On the relation between cardiodynamics and heartbeat perception. Psychophysiology 1993;30:467-74.

8 Van der Does AJW, Antony MM, Ehlers A, et al. Heartbeat perception in panic disorder: a reanalysis. Behav Res Ther 2000;38:47-62.

9 Khalsa SS, Rudrauf D, Feinstein JS, et al. The pathways of interoceptive awareness. Nat Neurosci 2009;12:1494-6.

10 Critchley HD, Wiens S, Rotshtein P, et al. Neural systems supporting interoceptive awareness. Nat Neurosci 2004;7:189-95.

11 Pollatos O, Gramann K, Schandry R. Neural systems connecting interoceptive awareness and feelings. Hum Brain Mapp 2007;28:9-18.

12 Moon C-M, Yang J-C, Jeong G-W. Explicit verbal memory impairments associated with brain functional deficits and morphological alterations in patients with generalized anxiety disorder. J Affect Disord 2015;186:328-36.

13 Strawn JR, Wehry AM, Chu W-J, et al. Neuroanatomic abnormalities in adolescents with generalized anxiety disorder: a voxel-based morphometry study. Depress Anxiety 2013;30:842-8.

14 Molent C, Maggioni E, Cecchetto F, et al. Reduced cortical thickness and increased gyrification in generalized anxiety disorder: a $3 \mathrm{~T} \mathrm{MRI}$ study. Psychol Med 2018;48:2001-10.

15 Maron E, Nutt D. Biological markers of generalized anxiety disorder. Dialogues Clin Neurosci 2017;19:147-58.

16 AP A. The Diagnostic and Statistical Manual of Mental Disorders(DSM-IV. Washington, DC: 435 American Psychiatric Association, 1994.

17 First MB SR, Gibbon M, Williams JBW. Structured clinical interview for DSM-IV-TR axis I disorders, research version, patient edition. (SCID-I/P. New York: Biometrics Research, New York State Psychiatric Institute, 2002.

18 Hamilton MAX. The assessment of anxiety states by rating. Br J Med Psychol 1959;32:50-5.
19 Hamilton M. A rating scale for depression. J Neurol Neurosurg Psychiatry 1960;23:56-62.

20 Khalsa SS, Lapidus RC. Can Interoception improve the pragmatic search for biomarkers in psychiatry? Frontiers in Psychiatry 2016;7.

21 Schandry R. Heart beat perception and emotional experience. Psychophysiology 1981;18:483-8.

22 Pollatos O, Herbert BM, Kaufmann C, et al. Interoceptive awareness, anxiety and cardiovascular reactivity to isometric exercise. Int $J$ Psychophysiol 2007;65:167-73.

23 Good CD, Johnsrude IS, Ashburner J, et al. A voxel-based morphometric study of ageing in 465 normal adult human brains. Neuroimage 2001;14:21-36.

24 Maldjian JA, Laurienti PJ, Kraft RA, et al. An automated method for Neuroanatomic and cytoarchitectonic atlas-based interrogation of fMRI data sets. Neuroimage 2003;19:1233-9.

25 Pang J, Tang X, Li H, et al. Altered interoceptive processing in generalized anxiety Disorder-A Heartbeat-Evoked potential research. Front Psychiatry 2019;10:616.

26 Andreescu C, Gross JJ, Lenze E, et al. Altered cerebral blood flow patterns associated with pathologic worry in the elderly. Depress Anxiety 2011;28:202-9.

27 Greenberg T, Carlson JM, Cha J, et al. Ventromedial prefrontal cortex reactivity is altered in generalized anxiety disorder during fear generalization. Depress Anxiety 2013;30:242-50.

28 Chang C-hui, Grace AA. Inhibitory modulation of orbitofrontal cortex on medial prefrontal Cortex-Amygdala information flow. Cereb Cortex 2018;28:1-8.

29 Kujawa A, Swain JE, Hanna GL, et al. Prefrontal reactivity to social signals of threat as a predictor of treatment response in anxious youth. Neuropsychopharmacol 2016;41:1983-90.

30 Terasawa Y, Shibata M, Moriguchi Y, et al. Anterior insular cortex mediates bodily sensibility and social anxiety. Soc Cogn Affect Neurosci 2013;8:259-66.

31 Zaki J, Davis JI, Ochsner KN. Overlapping activity in anterior insula during interoception and emotional experience. Neuroimage 2012;62:493-9.

32 Terasawa $\mathrm{Y}$, Kurosaki $\mathrm{Y}$, Ibata $\mathrm{Y}$, et al. Attenuated sensitivity to the emotions of others by insular lesion. Front Psychol 2015;6:1314.

33 Shang J, Fu Y, Ren Z, et al. The common traits of the ACC and pFc in anxiety disorders in the DSM-5: meta-analysis of voxel-based morphometry studies. PLoS One 2014;9:e93432.

34 Terlevic R, Isola M, Ragogna M, et al. Decreased hypothalamus volumes in generalized anxiety disorder but not in panic disorder. $J$ Affect Disord 2013;146:390-4.

35 Makovac E, Meeten F, Watson DR, et al. Neurostructural abnormalities associated with axes of emotion dysregulation in generalized anxiety. Neuroimage Clin 2016;10:172-81.

36 Liao M, Yang F, Zhang Y, et al. Childhood maltreatment is associated with larger left thalamic gray matter volume in adolescents with generalized anxiety disorder. PLoS One 2013;8:e71898.

37 Moon C-M, Jeong G-W. Functional and morphological alterations associated with working memory dysfunction in patients with generalized anxiety disorder. Acta radiol 2017;58:344-52.

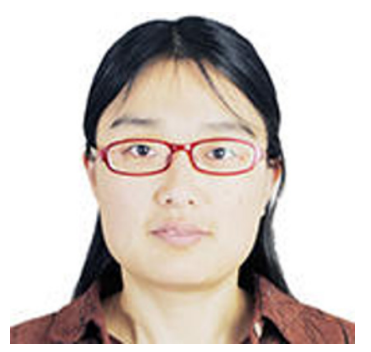

Hui Li received a bachelor's degree from the Department of Clinical Medicine of Hebei Medical University in 2003. She obtained the doctor's degree from Shanghai Jiao Tong University of Medicine in 2013. She visited the Nottingham University as research fellow in 2013 and visited Mayo Clinic as a visiting clinician in July 2017. Since July 2007, she worked as a psychiatrist in the Shanghai Mental Health Center(SMHC), Shanghai Jiao Tong University, School of Medicine. She is currently working as an attending doctor in the Department of Clinical Research Center of SMHC. Her research interests include the etiology and clinical research of anxiety disorder. 
Correction: Altered heartbeat perception sensitivity associated with brain structural alterations in generalised anxiety disorder

Li H, Zhang B, Hu $\mathrm{Q}$ et al. Altered heartbeat perception sensitivity associated with brain structural alterations in generalised anxiety disorder. General Psychiatry 2020;33:e100057. doi: 10.1136/gpsych-2019-100057.

The following sections contained errors and have been amended:

1. Figure 1 should be displayed as follows:

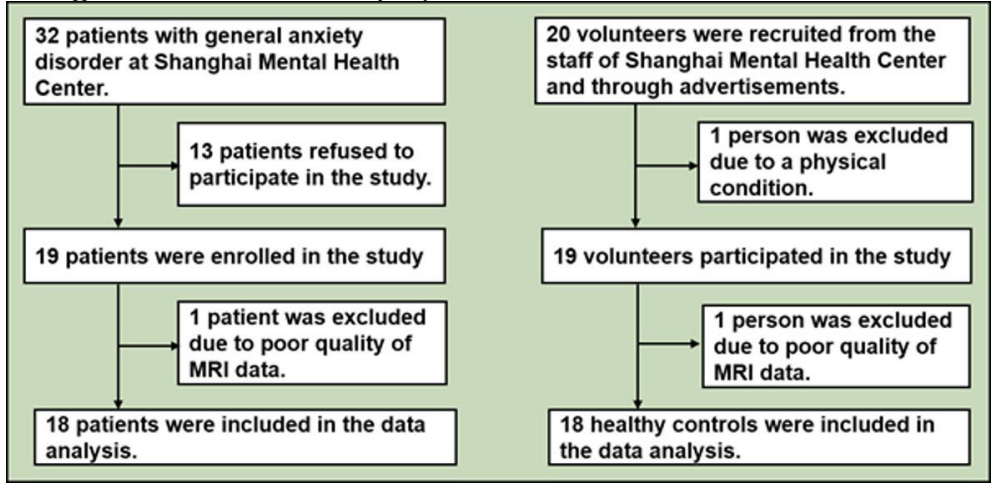

2. In Figure 3, axis x should be HPS, not HRS.

3. In the final paragraph of the main findings section, the following sentence should be: "In this current study, we investigated regional GMV in patients with GAD in a priori region from well-developed neuroanatomical theories of heartbeat perception. 'In' has replaced 'and applied'.

4. The author biography should be listed as follows:

Hui Li received a bachelor's degree from the Department of Clinical Medicine of Hebei Medical University in 2003. She obtained a doctorate from Shanghai Jiao Tong University of Medicine in 2013. She visited Nottingham University as a research fellow in 2013 and visited the Mayo Clinic as a visiting clinician in July 2017. Since July 2007, she has worked as a psychiatrist at the Shanghai Mental Health Centre, Shanghai Jiao Tong University School of Medicine. She is currently working as an attending doctor in the Department of Clinical Research Centre of SMHC. Her research interests include the aetiology and clinical research of anxiety disorders.

\section{() \\ OPEN ACCESS}

Open access This is an open access article distributed in accordance with the Creative Commons Attribution Non Commercial (CC BY-NC 4.0) license, which permits others to distribute, remix, adapt, build upon this work non-commercially, and license their derivative works on different terms, provided the original work is properly cited, appropriate credit is given, any changes made indicated, and the use is non-commercial. See: http://creativecommons.org/licenses/by-nc/4.0/.

(C) Author(s) (or their employer(s)) 2020. Re-use permitted under CC BY-NC. No commercial re-use. See rights and permissions. Published by BMJ.

General Psychiatry 2020;33:e100057corr1. doi:10.1136/gpsych-2019-100057corr1

Check for updates 